\title{
Utilización de cenizas volantes y mineralizadores como materia prima en la fabricación de cemento
}

\author{
Dra. TERESA BLANCO VARELA, Dr. TOMAS VAZQUEZ MORENO y Lcdo. ANGEL PALOMO SANCHEZ
}

$R E S U M E N$

En el presente trabajo se estudia el proceso de formación de un clinker de bajo consumo energético, empleando como materia prima cenizas volantes sulfocálcicas (en sustitución parcial del crudo tradicional) y fluorita.

Se demuestra la validez de dichas cenizas volantes, obteniendo clínker a $1.340^{\circ} \mathrm{C}$. Se estudia la composición mineralógica, capacidad de mineralización y temperatura de formación de la nueva fase líquida.

\section{$S U M M A R Y$}

In this research the process of formation of a low energy clinker is studied, when fly-ashes (rich in sulphates and lime) and fluorspar are used as raw materials (fly-ashes replaces to the tradicional raw mix partially).

The availability of those fly-ashes is shown since clinker at $1.340^{\circ} \mathrm{C}$ is obtained. The mineralogical composition, mineralizer capacity and temperature of formation of the new liquid phase is studied.

\section{IN T R O D U C CION}

El proceso de clinkerización consume más del $90 \%$ de la energía total necesaria para la fabricación del cemento.

A su vez la reacción de descarbonatación del $\mathrm{CaCO}_{3}$, para obtener $\mathrm{CaO}$, es la etapa que precisa de mayor energía dentro de las que se suceden en la cocción del clínker.

Las fases tradicionales del clínker portland $\left(\mathrm{C}_{3} \mathrm{~S}, \mathrm{C}_{2} \mathrm{~S}, \mathrm{C}_{3} \mathrm{~A}, \mathrm{C}_{4} \mathrm{AF}\right)^{*}$ están constituidas, todas ellas, por un alto porcentaje de $\mathrm{CaO}$. Los compuestos $\mathrm{C}_{3} \mathrm{~S}$ "alita" y $\mathrm{C}_{2} \mathrm{~S}$ "belita" producen en su hidratación la llamada "fase tobermorítica" que es un silicato hidratado de baja relación $\mathrm{C} / \mathrm{S}$ y que aporta la práctica totalidad de la resistencia mecánica (e incluso química) del cemento hidratado. $\mathrm{La}$ "fase líquida", $\mathrm{C}_{3} \mathrm{~A}$ y $\mathrm{C}_{4} \mathrm{AF}$, necesaria principalmente para la formación de alita $\mathrm{y}$ belita, apenas sí, en su hidratación aportan compuestos que contribuyan en el total de las resistencias resultantes en la hidratación del cemento.

En un trabajo anterior (1) se abordó el ahorro de energia en la clinkerización en función de una sustitución parcial de las fases alumínica y ferritica, por una fase del tipo fluorellestadita, $3 \mathrm{C}_{2} \mathrm{~S} .3 \mathrm{CaSO}_{4} \cdot \mathrm{CaF}_{2}$. Para ello sería necesario introducir en el crudo minerales tales como fluorita $\mathrm{y}$ anhidrita.

Por otra parte, las cenizas volantes, que son un subproducto del quemado del carbón en centrales térmicas constituyen -a pesar de su creciente utilización industrial- una amenaza ecológica $\mathrm{y}$, en cierto modo, un derroche ecomómico. La producción de cenizas volantes en España es, en la actualidad de unas $9.000 .000 \mathrm{t}$ al año.

* Se emplea la notacion admitida en la qumicia del cemento: $\mathrm{C}=\mathrm{CaO}: \mathrm{A}=\mathrm{Al}_{2} \mathrm{O}_{3}: F=\mathrm{Fe}_{2} \mathrm{O}_{3}: \mathrm{S}=\mathrm{SiO}_{2}$. 
Según sea su naturaleza química se podrían clasificar en dos grandes grupos: silicoaluminosas y sulfocálcicas. El primer grupo (silicoaluminosas) contienen un elevado procentaje de $\mathrm{SiO}_{2}$ y $\mathrm{Al}_{2} \mathrm{O}_{3}$. Las sulfocálcicas lo contienen de $\mathrm{CaO}$ libre y de $\mathrm{SO}_{3}$. La producción española de este tipo de cenizas es, aproximadamente, de $1.000 .000 \mathrm{t}$ anuales. La observación microscópica de estas cenizas revela que están formadas por cenosferas (materia vitrea de silicoaluminatos y ferritos), cristales de $\mathrm{Ca}(\mathrm{OH})_{2}, \mathrm{CaO}, \mathrm{CaSO}_{4}$ y partículas de carbón.

En el cuadro 1 se dan los análisis de algunas cenizas volantes silicoaluminosas o sulfocálcicas, procedentes de térmicas españolas.

\begin{tabular}{|c|c|c|c|c|c|}
\hline \multicolumn{6}{|c|}{$\begin{array}{cc}\text { C U A D RO } & 1 \\
\text { Análisis de cenizas volantes }\end{array}$} \\
\hline & \multicolumn{3}{|c|}{ Silicoaluminosas } & \multicolumn{2}{|c|}{ Sulfocálcicas } \\
\hline & 1 & 2 & 3 & 4 & 5 \\
\hline P.F. & 5,7 & 2,9 & 0,8 & 3,8 & 6,6 \\
\hline R.I. & 0,1 & 0,1 & 0,1 & 0,1 & 0,1 \\
\hline $\mathrm{SiO}_{2}$ & 51,2 & 45,0 & 45.4 & 12,6 & 14.1 \\
\hline $\mathrm{Al}_{2} \mathrm{O}_{3}$ & 30,8 & 36,7 & 30,0 & 7,6 & 8.2 \\
\hline $\mathrm{Fe}_{2} \mathrm{O}_{3}$ & 6,4 & 7.4 & 16,1 & 2.5 & 3,0 \\
\hline $\mathrm{CaO}$ & 3,0 & 4,4 & 4,0 & 60,2 & 55,0 \\
\hline $\mathrm{MgO}$ & 1,9 & 1.3 & 1,1 & 1,0 & 1,9 \\
\hline $\mathrm{SO}_{3}$ & 0,4 & 1,4 & 1,9 & 11,6 & 11,1 \\
\hline
\end{tabular}

A la vista de los análisis quimicos de las cenizas volantes, se observa que en su composición están los óxidos que son necesarios para la obtención del clínker tradicional. Es decir, podrían sustituir total o parcialmente la caliza y arcilla como crudos del cemento portland.

En el caso de las cenizas de naturaleza sulfocálcica, se pueden deducir una serie de consideraciones:

- al aportar $\mathrm{CaO}$ pueden hacer más reducida la necesaria inclusión de caliza al crudo, con el ahorro energético importante que supone el evitar la descarbonatación del $\mathrm{CaCO}_{3}$ :

- al aportar $\mathrm{SO}_{3}$, parecen idóneas para conseguir una fase líquida como la propuesta en (1), energéticamente barata;

- al contener algo de carbón, que se quemará en el proceso de clinkerización, se obtendrá un ahorro de energia adicional al expuesto;

- su naturaleza quimica, principalmente por el elevado contenido en $\mathrm{CaO}$, hace que este tipo de cenizas no sea suficientemente adecuado para su empleo como adición al cemento portland, una de las utilizaciones más extendidas de las cenizas volantes.

El empleo de fundentes (compuestos que hacen descender el punto de fusión de la fase liquida) y/o de mineralizadores (compuestos que aceleran la velocidad de un proceso o reacción que tiene lugar en estado sólido, dentro de la fase líquida, o en la interfase sólido-liquido) ha sido 
contemplado como medio de ahorrar energía en la clínkerización. El mineralizador y fundente más conocido es el $\mathrm{CaF}_{2}$. Se ha estudiado desde diversos aspectos, tales como la relación entre su electronegatividad y la viscosidad de la fase líquida (2) y (7); el aumento de la reactividad de esta fase con la proporción en la que está presente (3); la variación de la velocidad de reacciones de clinkerización (4); la disminución de la temperatura de formación del clínker (5), etc....

Otro mineralizador empleado, aunque menos frecuentemente que el $\mathrm{CaF}_{2}$, es el $\mathrm{CaSO}_{4}$. Su efecto no es aún suficientemente conocido, existiendo resultados no concordantes entre algunos investigadores. En todo caso parece probada su capacidad de disolución en los silicatos bi y tricálcicos. Asi, el $\mathrm{C}_{2} \mathrm{~S}$ obtenido en presencia de $\mathrm{CaSO}_{4}$ se encuentra a temperatura ambiente en forma $\beta$ o $\alpha$, en función de la proporción del $\mathrm{CaSO}_{4}$ y del tratamiento térmico aplicado. Tanto una como otra forma contiene $\mathrm{CaSO}_{4}$ en disolución sólida, resultando con unas alteraciones estructurales que potenciarian su hidraulicidad.

La bibliografía consultada da escasas referencias sobre las fases existentes a altas temperaturas en el sistema $\mathrm{CaO}-\mathrm{SiO}_{2}-\mathrm{Al}_{2} \mathrm{O}_{3}-\mathrm{CaSO}_{4}-\mathrm{CaF}_{2}-\mathrm{Fe}_{2} \mathrm{O}_{3}$. En el IETcc el efecto conjunto de ambos mineralizadores $\mathrm{CaF}_{2}$ y $\mathrm{CaSO}_{4}$, ha sido estudiado por Blanco-Varela, que ha desarrollado su Tésis Doctoral en ese Tema (6).

\section{OBJETO DEL TRABAJO}

En el presente trabajo se estudia el proceso de formación de un nuevo clinker, con un bajo consumo energético, empleando como crudos cenizas volantes de naturaleza sulfocálcica -que sustituirán parcialmente a los crudos tradicionales-, y fluorita; se estudia la formación de una nueva fase liquida de bajo coste de energia, que sustituye parcialmente a la fase líquida $\left(\mathrm{C}_{3} \mathrm{~A}\right.$ y $\mathrm{C}_{4} \mathrm{AF}$ ) existente en el clinker portland tradicional.

\section{PARTE EXPERIMENTAL}

\section{Material y métodos}

Se dosificó un crudo a partir de una ceniza volante, procedente de una Central Térmica española, y $\mathrm{CaCO}_{3}, \mathrm{SiO}_{2}, \mathrm{Al}_{2} \mathrm{O}_{3}, \mathrm{CaF}_{2}$ y $\mathrm{Fe}_{2} \mathrm{O}_{3}$ (R. A. de Merck). Los análisis de la ceniza volante de partida y del crudo dosificado se dan en el cuadro 2.

La mezcla de estos materiales se homogeneizó en una mezcladora Turbula durante 1 hora y, posteriormente se molió en medio líquido (utilizando acetona) en molino de ágata mecánico.

El comportamiento térmico de esta muestra se estudió por AT, y el clínker obtenido se examinó por DRX y espectroscopia IR.

Se hicieron pastillas de este crudo en una prensa y a una presión de, aproximadamente, $400 \mathrm{kp} / \mathrm{cm}^{2}$; el diámetro de las pastillas fue de $2 \mathrm{~cm}$ y el peso aproximado de 2 gramos.

El crudo así preparado se clinkerizó a 3 temperaturas diferentes $1.125^{\circ} \mathrm{C}, 1.187^{\circ} \mathrm{C}$ y $1.337^{\circ} \mathrm{C}$ (temperaturas obtenidas de los resultados aportados por el ATD) por espacio de 30 minutos.

Los clinkeres obtenidos se estudiaron por DRX y espectroscopia IR.

Se efectuó el ataque de estos clínkeres con metanol-salicilico [método de Takashima (8)] para disolver los silicatos y estudiar por medio de las técnicas ya mencionadas, la fase líquida que poseen. 
Se evaluó la aptitud a la cocción de los clínkeres obtenidos por medio del análisis del $\mathrm{CaO}$ no combinado, por el método del etilen-glicol (9).

\section{CUADRO 2}

Análisis químico de la ceniza y del crudo

\begin{tabular}{|l|c|c|}
\cline { 2 - 3 } \multicolumn{1}{c|}{} & Ceniza V. de partida & Crudo dosificado \\
\cline { 2 - 3 } & \% & \% \\
\hline P.F. & 9,46 & 32,38 \\
$\mathrm{R} . \mathrm{I}$. & 0,02 & \\
$\mathrm{SiO}_{2}$ & 11,85 & 16,15 \\
$\mathrm{Al}_{2} \mathrm{O}_{3}$ & 7,34 & 1,93 \\
$\mathrm{Fe}_{2} \mathrm{O}_{3}$ & 2.93 & 1,21 \\
$\mathrm{CaO}^{1}$ & 57.35 & 46,04 \\
$\mathrm{MgO}_{\mathrm{SO}}$ & 0,93 & 0,14 \\
$\mathrm{SO}_{3}$ & 10,54 & 1,62 \\
$\mathrm{Na}_{2} \mathrm{O}$ & 0,10 & 0,01 \\
$\mathrm{~K}_{2} \mathrm{O}$ & 0,38 & 0,01 \\
$\mathrm{CaF}_{2}$ & & 0,52 \\
\hline
\end{tabular}

\section{RES ULT A D O S}

La curva de ATD del crudo se da en la fig. 1, y presenta hasta la temperatura de $1.000^{\circ} \mathrm{C}$, las señales térmicas características de un crudo de clínker portland, si bien la descarbonatación es menos intensa por ser la ceniza volante la que aporta parte del óxido cálcico necesario en la dosificación y no estar éste en forma de carbonato.

A partir de esta temperatura y hasta la de $1.450^{\circ} \mathrm{C}$, la curva de ATD presenta dos picos endotérmicos: $\mathrm{El}$ primero a $1.175^{\circ} \mathrm{C}$ que, como se verá más adelante se produce por la descomposición del $3 \mathrm{C}_{2} \mathrm{~S} .3 \mathrm{CaSO}_{4} \cdot \mathrm{CaF}_{2}$ y el segundo a $1.305^{\circ} \mathrm{C}$, se debe a la fusión de ferritos $\mathrm{y}$ aluminatos.

Hacia $1.210^{\circ} \mathrm{C}$ existe una pequeña y ancha señal endotérmica que se interpreta que es debida a la transformación $\beta \longrightarrow \alpha$ del $\mathrm{CaSO}_{4}$.

En el enfriamiento se ven dos picos exotérmicos solapados a 1.205 y $1.187^{\circ} \mathrm{C}$ debidos, respectivamente, a la solidificación de la fase líquida y a la transformación $\alpha \longrightarrow \beta$ del $\mathrm{CaSO}_{4}$.

A la temperatura de $1.075^{\circ} \mathrm{C}$ hay otro pico exotérmico de menor tamaño. Para conocer la naturaleza de este pico se realizó un nuevo ATD hasta la temperatura de $1.250^{\circ} \mathrm{C}$ (posterior al primero de los picos endotérmicos). En el enfriamiento aparece un único pico exotérmico a $1.075^{\circ} \mathrm{C}$, y que es inverso al endoteŕmico presente en la curva de calentamiento. Asi pues se trata de un proceso reversible, que como ya hemos dicho se debe a la descomposición del $3 \mathrm{C}_{2} \mathrm{~S} .3 \mathrm{CaSO}_{4} \cdot \mathrm{CaF}_{2}$ y su formación de nuevo en el enfriamiento. 
Es de destacar que en la curva TG de la fig. 1, no se observan pérdidas de masa posteriores a la descarbonatación hasta la temperatura de $1.360^{\circ} \mathrm{C}$.

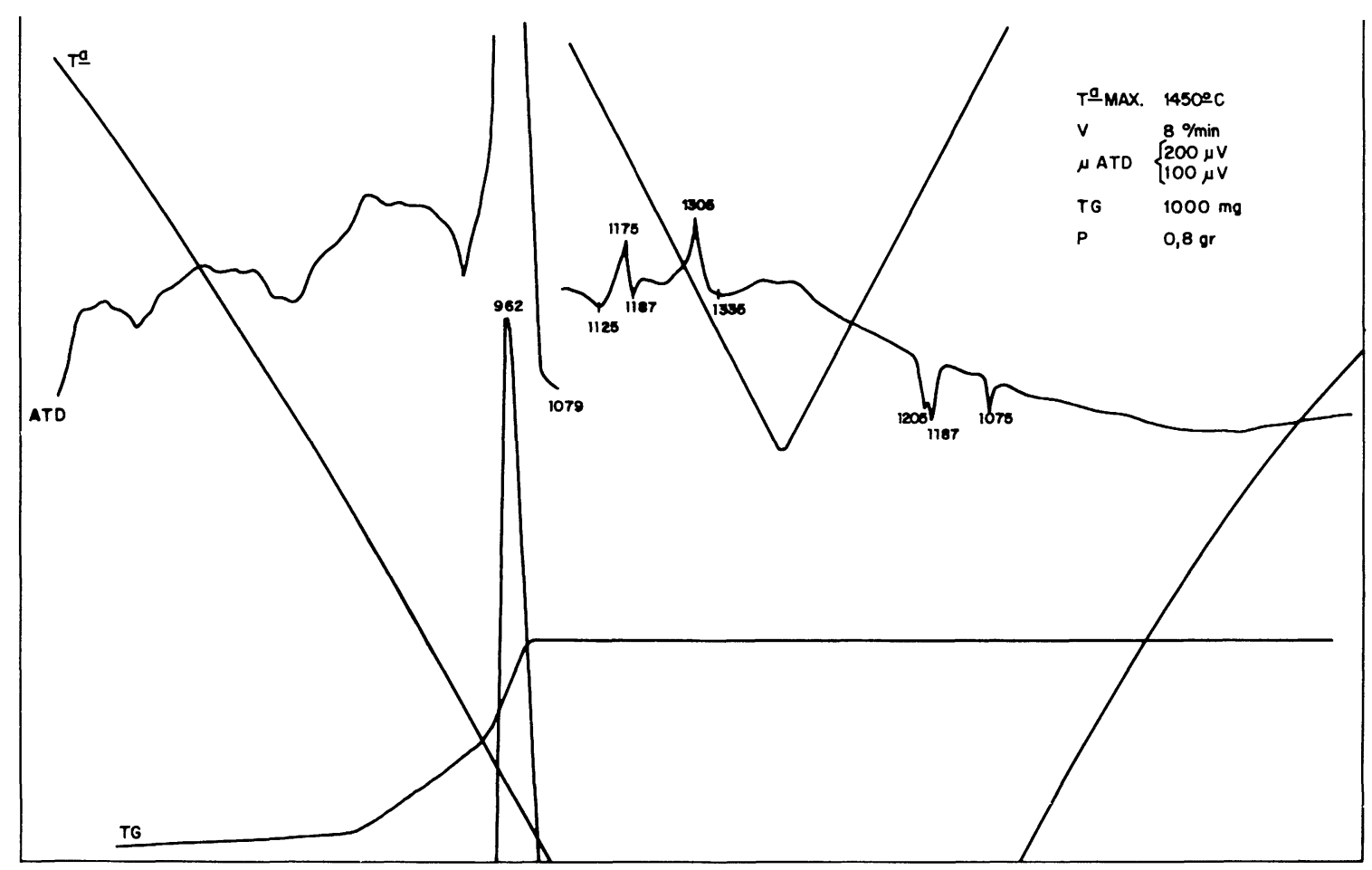

Fig. 1

El espectro IR del clínker obtenido en el horno del ATD a la temperatura máxima de $1.450^{\circ} \mathrm{C}$, se corresponde con el de un clinker tradicional que contenga sulfatos.

El difractograma de $\mathrm{RX}$ es el de un clinker tradicional con abundante alita, algo de belita, ferritos y aluminatos en proporción algo inferior y posiblemente $\mathrm{CaSO}_{4}$ y fluorellestadita.

Para conocer la naturaleza de las señales endotérmicas de la curva de ATD y descubrir los caminos por los que transcurre la clinkerización de los crudos fabricados con cenizas volantes y mineralizadores, se realizaron cocciones de las muestras durante 30 minutos a las temperaturas de $1.125,1.187$ y $1.337^{\circ} \mathrm{C}$. Las muestras fueron templadas al aire.

El cuadro 3 resume la composición de estas muestras según datos aportados por los difractogramas realizados sobre los clínkeres y sobre el residuo del ataque del método de Takashima a los mismos.

Los espectros de IR de estos clínkeres se dan en las figs. 2 y 3.

El contenido en $\mathrm{CaO}$ de estos clinkeres valorado por el método del etilen-glicol fue:

Clinker templado a $1.125^{\circ} \mathrm{C} \ldots \ldots \ldots \ldots \ldots \ldots \ldots \ldots \ldots$

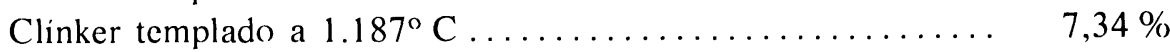

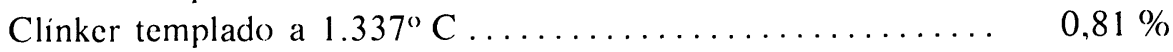


C UADRO 3

Composición de las muestras según los datos aportados por los difractogramas

\begin{tabular}{|c|c|c|c|c|c|c|c|c|c|}
\hline $\mathbf{T}^{0} \mathbf{C}$ & $\mathbf{C a O}$ & $\mathbf{C}_{3} \mathbf{S}$ & $\mathbf{C}_{2} \mathbf{S}$ & $\mathbf{F l}(\mathbf{1})$ & $\mathbf{C a S O}_{4}$ & $\begin{array}{c}\mathbf{C}_{12} \mathbf{A}_{7} \\
\mathbf{C}_{11} \mathbf{A}_{7} \\
. \mathbf{C a}_{2}\end{array}$ & $\mathbf{C}_{4} \mathbf{A F}$ & $\begin{array}{c}3 \mathbf{C A} . \\
. \mathbf{C a S O}_{4} \\
(2)\end{array}$ & $\mathbf{C}_{3} \mathbf{A}$ \\
\hline 1.125 & $* *$ & $*$ & $* * *$ & $*$ & - & $*$ & $*$ & - & - \\
1.187 & $*$ & $* *$ & $* *$ & - & $*$ & $*$ & $*$ & $*$ & - \\
1.337 & - & $* * *$ & $*$ & - & Trazas & $*$ & $*$ & - & $*$ \\
\hline
\end{tabular}

(1) $\mathrm{Fl}=$ Fluorellestadita. (2) Similar al, del tipo de.

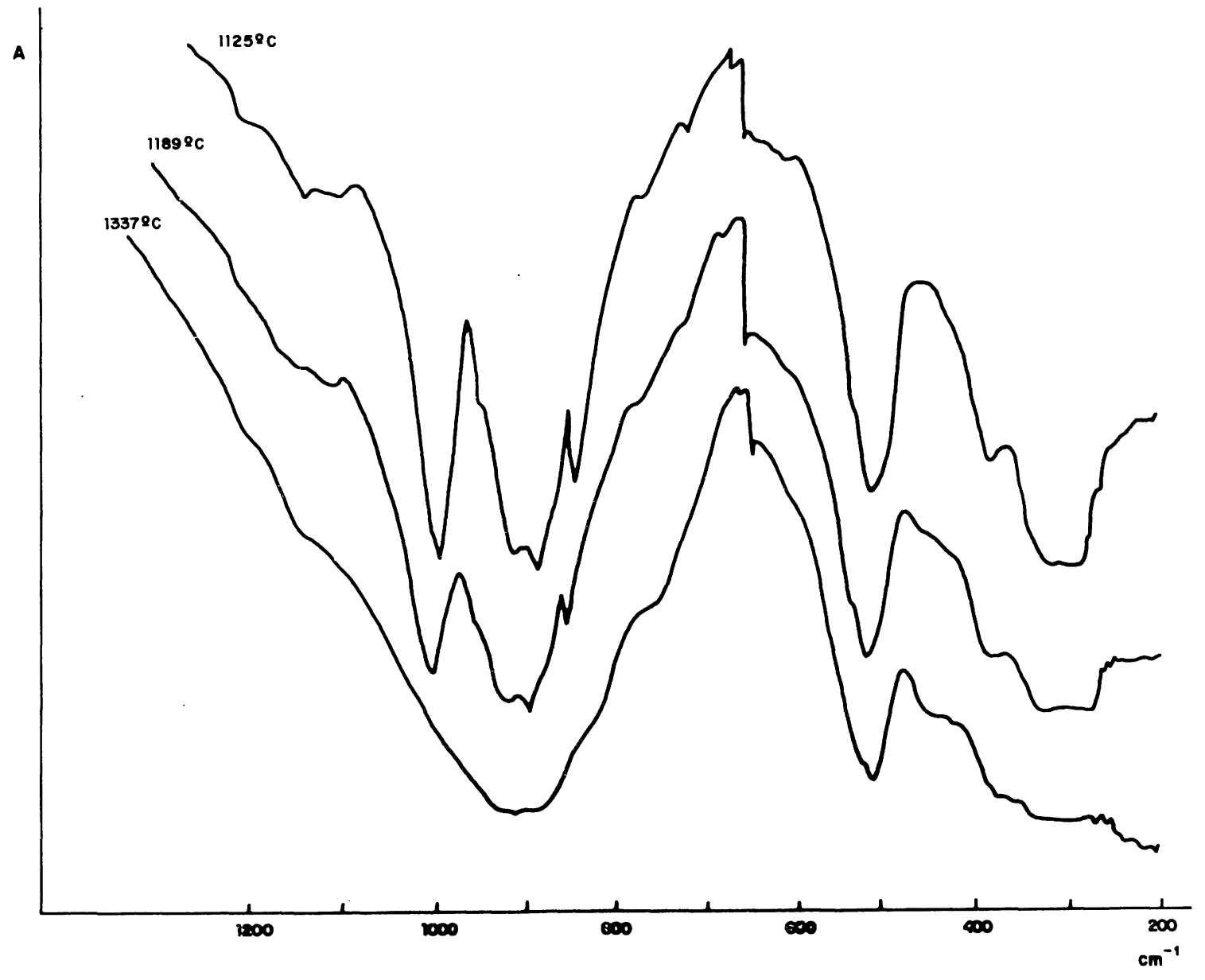

Fig. 2

\section{S C US I O N}

Examinando los resultados descritos en los análisis de $\mathrm{RX}$ y $\mathrm{CaO}$ libre de los clínkeres templados a las temperaturas de $1.125^{\circ} \mathrm{C}, 1.187^{\circ} \mathrm{C}$ y $1.337^{\circ} \mathrm{C}$, se extraen importantes datos sobre la evolución del clinker en general y de los silicatos en particular.

Asi se puede concluir lo siguiente:

- La cantidad de $\mathrm{CaO}$ libre presente en la muestra es muy inferior a la habitual en crudos comerciales clinkerizados a estas temperaturas (14). 


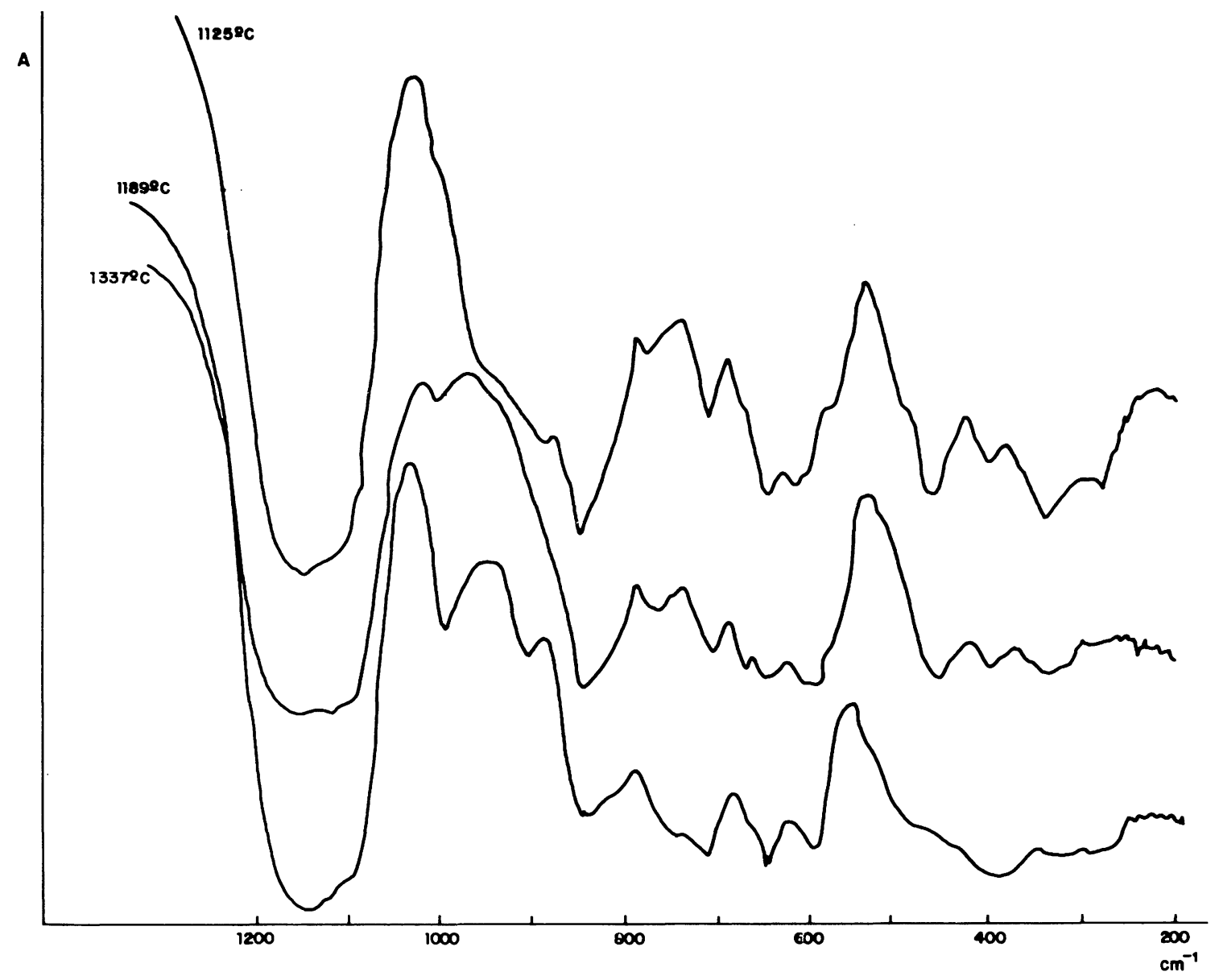

Fig. 3

Esta diferencia se acusa más a mayores temperaturas, dado que la temperatura de $1.125^{\circ} \mathrm{C}$ la cantidad de $\mathrm{CaO}$ libre es entre 2 y 2,5 veces inferior y a la de $1.337^{\circ} \mathrm{C}$ de 3 a 4 veces inferior.

Por consiguiente las muestras se presentan a todas las temperaturas mucho más evolucionadas conteniendo una mayor proporción de silicatos. A la temperatura de $1.125^{\circ} \mathrm{C}$ ya se encuentra $\mathrm{C}_{3} \mathrm{~S}$ en una proporción considerable $(=10 \%)$, lo que indica que en presencia de $\mathrm{F}^{-} \mathrm{y}$ $\mathrm{SO}_{4}^{=}$el campo de estabilidad del $\mathrm{C}_{3} \mathrm{~S}$ se amplia. Gutt y Osborne (11) afirman que el campo de estabilidad del $\mathrm{C}_{3} \mathrm{~S}$ aumenta con la presencia de $\mathrm{F}^{-}$en el sistema, estando su límite inferior en $1.170^{\circ} \mathrm{C}$. Mukerjii (12) estudió el sistema $\mathrm{C}-\mathrm{S}-\mathrm{CaF}_{2}$ y dió más amplitud al campo de estabilidad del $\mathrm{C}_{3} \mathrm{~S}$ que el establecido por Eitel (13) extendiéndolo hasta $1.107^{\circ} \mathrm{C}$, aunque este autor no tiene en cuenta la existencia del $3 \mathrm{C}_{3} \mathrm{~S} \mathrm{CaF}_{2}$.

Tanto la abundancia de silicato tricálcico a temperaturas por debajo de su campo de estabilidad como el estado tan avanzado de las reacciones a tan bajas temperaturas descrito en los dos párrafos anteriores, encuentran su justificación en los dos hechos siguientes:

- Capacidades mineralizadoras y fundentes de $\operatorname{los} \mathrm{F}^{-}$y $\mathrm{SO}_{4}^{=}$empleados en sustitución parcial de los ferritos y aluminatos.

- Estado amorfo inicial de las cenizas volantes.

El espectro de la muestra tratada a $1.125^{\circ} \mathrm{C}$ da las bandas características del $\beta-\mathrm{C}_{2} \mathrm{~S}$, muy bien cristalizado; en el espectro de la muestra tratada a $1.189^{\circ} \mathrm{C}$ se dan así mismo, las bandas del 
$\beta-C_{2} S$, pero con una peor definición, que se interpreta como una disminución en el orden estructural. Ambas muestras contienen la fase ferritica probablemente del tipo $\mathrm{C}_{4} \mathrm{AF}$, y los aluminatos como $\mathrm{C}_{12} \mathrm{~A}_{7}$, o bien $\mathrm{C}_{11} \mathrm{~A}_{7} \cdot \mathrm{CaF}_{2}$.

La muestra de $1.125^{\circ} \mathrm{C}$ contiene fluorellestadita. Su presencia en la de $1.189^{\circ} \mathrm{C}$ es dudosa. Sin embargo en su espectro hay bandas que se asignan a una fase del tipo $3 \mathrm{CA}^{-\mathrm{CaSO}_{4}}$. El espectro de la muestra tratada a $1.337^{\circ} \mathrm{C}$ presenta esas bandas con mayor intensidad: las caracteristicas de la fluorellestadita no se observan. En esta muestra, la fase $\mathrm{C}_{12} \mathrm{~A}_{7}$ (ó $\mathrm{C}_{11} \mathrm{~A}_{7} \cdot \mathrm{CaF}_{2}$ ) también se encuentra. Además, aparecen bandas que demuestran la presencia de $C_{3} A$. Lo más característico del espectro de esta muestra son las bandas de $\mathrm{C}_{3} \mathrm{~S}$, se encuentra en gran proporción y con gran desorden estructural, lo que indica una probable reactividad muy acusada.

La desaparición de las bandas características de la fluorellestadita, con una simultánea aparición de bandas que se interpreten como de una fase cercana a $3 \mathrm{CA}^{-\mathrm{CaSO}_{4}}$ se explica del modo siguiente: $\mathrm{El} 3 \mathrm{C}_{2} \mathrm{~S} .3 \mathrm{CaSO}_{4} \cdot \mathrm{CaF}_{2}$ puro se descompone con fusión a $1.260^{\circ} \mathrm{C}$, pero cuando se encuentra en un sistema más complejo, como el estudiado, a $1.189^{\circ} \mathrm{C}$ ha sufrido ya una fusión incongruente, dando una fase líquida de naturaleza fluosulfática y silicato bicálcico. A esa misma temperatura, los aluminatos presentes reaccionan con aquella fase sulfática para formar el $3 \mathrm{CA} \mathrm{CaSO}_{4}$ muy probablemente con $\mathrm{F}^{-}$y $\mathrm{SiO}_{2}$ en su red, en el transcurso del enfriamiento. Este compuesto posee una presión de vapor inferior a la del $\mathrm{CaSO}_{4}$, ya que las pérdidas de $\mathrm{SO}_{3}$ con el aumento de temperatura es treinta veces inferior en el citado $3 \mathrm{CA}^{-\mathrm{CaSO}_{4}}$ que en el $\mathrm{CaSO}_{4}$.

El comportamiento térmico de un crudo tradicional es muy diferente de el del crudo objeto de este estudio. Dejando aparte las conocidas diferencias de un crudo que tenga una descarbonatación menor por aportar el $\mathrm{CaO}$ en forma de $\mathrm{Ca}(\mathrm{OH})_{2}$ o combinado con silicatos y aluminatos y no como $\mathrm{CaCO}_{3}$ es, a temperaturas superiores a $\operatorname{los} 1.000^{\circ} \mathrm{C}$ donde las divergencias son más marcadas.

La curva de ATD de un crudo Portland presenta hacia $1.250^{\circ} \mathrm{C}$ un fuerte pico exotérmico de reacción que se debe preferentemente a la reacción del $\mathrm{C}_{2} \mathrm{~S}$ con $\mathrm{CaO}$ para dar $\mathrm{C}_{3} \mathrm{~S}$ y descomposición de ghelenita para dar $\mathrm{C}_{3} \mathrm{~A}$ y $\mathrm{C}_{2} \mathrm{~S}$. Es a partir de esta temperatura cuando se comienza la formación del $\mathrm{C}_{3} \mathrm{~S}$ en estos clínkeres (13).

Las curvas de ATD de la fig. 1, no presentan las mismas caracteristicas conteniendo los dos picos endotérmicos descritos en los resultados.

Las reacciones por las que transcurre la clinkerización, en este caso son las siguientes:

A la temperatura de $1.125^{\circ} \mathrm{C}$ existe ya $\mathrm{C}_{3} \mathrm{~S}$ en proporción de, aproximadamente un $10 \%, \mathrm{C}_{2} \mathrm{~S}$, $\mathrm{CaO}, \mathrm{C}_{4} \mathrm{AF}$, fluorellestadita y $\mathrm{C}_{12} \mathrm{~A}_{7}$ ó $\mathrm{C}_{11} \mathrm{~A}_{7} \cdot \mathrm{CaF}_{2}$. A esta temperatura no se encuentra $\mathrm{C}_{2} \mathrm{AS}$ cuya formación está inhibida por la presencia de $\mathrm{CaF}_{2}(13)$.

Como se ha dicho, a la temperatura de $1.175^{\circ} \mathrm{C}$ (primer pico endotérmico de ATD) se produce la descomposición de la fluorellestadita y posiblemente, también la formación de la fase parecida al $3 \mathrm{CA} \cdot \mathrm{CaSO}_{4}$.

Asi, a $1.187^{\circ} \mathrm{C}$ los clinkeres están constituidos por $\mathrm{C}_{3} \mathrm{~S}(\simeq 35 \%), \mathrm{C}_{2} \mathrm{~S}, \mathrm{CaO}, \mathrm{C}_{4} \mathrm{AF}, \mathrm{C}_{12} \mathrm{~A}_{7}$ ó $\mathrm{C}_{11} \mathrm{~A}_{7} \cdot \mathrm{CaF}_{2}, \mathrm{CaSO}_{4}$ y la fase parecida al $3 \mathrm{CA} \cdot \mathrm{CaSO}_{4}$.

Al aumentar la temperatura se produce a $1.305^{\circ} \mathrm{C}$ la fusión de ferritos y aluminatos y a la temperatura de $1.337^{\circ} \mathrm{C}$ las reacciones ya están finalizadas; el clinker está formado por $\mathrm{C}_{3} \mathrm{~S}$, $\mathrm{C}_{2} \mathrm{~S}, \mathrm{C}_{4} \mathrm{AF}$, algo de $\mathrm{C}_{3} \mathrm{~A}$ con $\mathrm{Na}_{2} \mathrm{O}$ en su estructura, $\mathrm{C}_{12} \mathrm{~A}_{7}$ o $\mathrm{C}_{11} \mathrm{~A}_{7} \cdot \mathrm{CaF}_{2}$ y el compuesto parecido a $3 \mathrm{CA} \cdot \mathrm{CaSO}_{4}$. 
Es de destacar que los picos de los dos últimos compuestos mencionados no se encuentran en los diagramas de RX y que su existencia está demostrada por los espectros de IR, lo que indica un pobre estado de cristalización.

\section{CON CLUSIONES}

1. ${ }^{a} \quad$ El campo se estabilidad del $\mathrm{C}_{3} \mathrm{~S}$ se ve sensiblemente aumentado por la presencia de $\mathrm{CaF}_{2}$ y $\mathrm{CaSO}_{4}$, encontrándose en las muestras en cantidad apreciable $(=10 \%)$, ya a la temperatura de $1.125^{\circ} \mathrm{C}$.

2. $\quad$ El empleo de $\mathrm{F}^{-}$y $\mathrm{SO}_{4}^{2}$, sustituyendo parcialmente a la fase liquida tradicional formada por aluminatos y ferritos demuestra tener una gran eficacia debido a:

- que se forman compuestos intermedios en el curso de la clinkerización, diferentes a los tradicionales, inhibiendose la formación de algunos de aquellos compuestos, dando lugar a la aparición de una fase liquida de naturaleza fluosulfática, a $1.175^{\circ} \mathrm{C}$.

Como consecuencia de ello, existe un aumento en la velocidad de formación de los silicatos cálcicos hidráulicamente activos.

3. A $\quad$ A la temperatura de $1.337^{\circ} \mathrm{C}$ el clinker ya está formado hacièndose innecesario el aumento de la temperatura para la obtencion de sus fases finales.

4. ${ }^{a} \quad$ En este tipo de clinkeres es desaconsejable que la temperatura de cocción supere los $1.360^{\circ} \mathrm{C}$, para evitar escapes de $\mathrm{F}^{-}$.

5. ${ }^{a}$ Se demuestra la validez de las cenizas volantes sulfocálcicas empleadas como materia prima en la fabricación de crudo de clinker con una fase liquida "no tradicional" y de bajo consumo energético.

\section{B I B L I O G R A F I A}

(1) BLANCO VARElA, T. y VAZQUEZ MORENO. T.: Materiales de Construcción n. ${ }^{\circ}$ 181. p. 55-64, (1981)

(2) PONOMAREV. I. F., GRACH`YAN, A. N. y ZUBERHIN, A. P.: Dokl. Akad. Nauk. SSSR., 166 (2) p. $410-412$ (1966).

(3) SHUBIN. V. I.: VI Congreso Internacional de la Quimica del Cemento, Moscú (1974).

(4) VOGEL, E.: Silikattechnik, 10 (8) 415 (1959).

(5) Vlasova. M. T.. JUdOVICH. B. E., ZHARKO, V. J., VOVCHOK. G. J. y BAZALEVSKIJ. V. R.: Cement: 3 , 9 (1979).

(6) BLANCO VARELA, T.: Tesis Doctoral, (octubre de 1980).

(7) TEOREANU. I.: Rev. Mat. Const., Trav. Publics., (666): p. 73-77 (1971).

(8) TAKASHIMA, S.: Semento Gijutzu Nenpo; XII, pp. 12-13, (1958).

(9) PLIEGO DE RECEPCION DE CONGLOMERANTES HIDRAULICOS: RC-75.

(10) BLANCO VARElA. T. y PALOMO SANCHEZ. A.: Cement and Concrete Research (en prensa).

(11) GRUTT. W. y OSBORNE, G. J.: Trans. Brit. Soc.. 69, 3, pp. 125-129 (1970).

Materiales de Construccion N. ${ }^{\circ} 189-1983$ 
(12) MUKERJI: Memories Scientifiques, Rev. Metallurg.. IX, N. ${ }^{\circ} 11,(1963)$.

(13) PALOMO SANCHEZ, A.: Memoria para aspirar al grado de Licendiado en Ciencias Quimicas; Universidad Complutense de Madrid, Facultad de Ciencias Quimicas; Madrid 1982.

(14) PALOMO SANCHEZ, A. y BLANCO VARELA. M. T.: Materiales de Construcción, N. ${ }^{\circ}$ 187. (1982).

\section{A GRA D ECIMIENTO}

Los autores desean expresar su agradecimiento al Grupo de Productores Nacionales de Minerales y Concentrados de Fluorita por la ayuda económica prestada a Angel Palomo, sin la cual no hubiera sido posible la realización de este trabajo.

\section{publicaciones del i.e.t.c.c.}

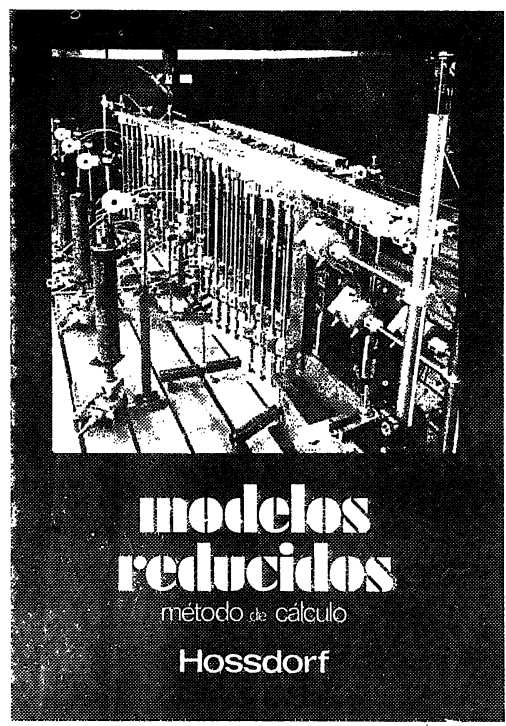

Modelos reducidos. Método de cálculo

H. Hossdorf, Ingeniero Civil

La técnica de los ensayos en modelos reducidos de estructuras sufre hoy dia una decisiva metamorfosis. estructuras sufre hoy dia una decisiva metamorfosis. Hasta hace poco era un medio más bien de artesa nia, que no siempre era tomado en serio for los academicos teorizantes para comprender el comportaque se acudió las más de las veces. como a un úlimo :emedio debido a sus indiscutibles insuficien. cias. Sin embargo, en poco tiempo y yracias a su conexión con los ordenadores digitales, se ha trans. formado en un instrumento cientificamente valioso que no puede quedar a un lado en la práctica diaria del ingeniero Proyectista.

Un volumen encuadernado en cartoné plastificado con lomo de tela, de $17 \times 24 \mathrm{~cm}$, compuesto dí 250 páginas, 153 figuras y fotografias.

Precios: 1.800 ptas.; \$ USA 26.00

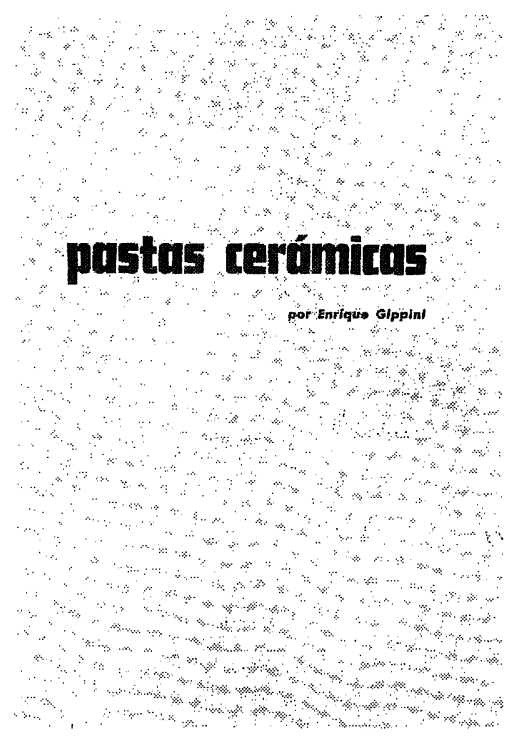

PASTAS CERAMICAS

Enrique Gippini,

Dr. en Ciencias Quimicas

El nexo de unión de todos los capitulos del libro es la idea subyacente de crear una teoria general de pastas. Moldeo y Cocción son los dos procesos a los que oebe adecuarse ia composición. Las caracteristicas isico-quimicas más importantes que deben presentar las pastas para que los resultados de estos procesos sean satisfactorios $y$ como pueden cambiar se dichas caracteristicas son los temas de discusió escogidos.

Un volumen encuadernado en cartoné, de $25 \times 17 \mathrm{~cm}$ compuesto de 259 páginas, 143 figuras y fotogra fi, $15, y 3$ tablas

Precios: 2.000 ptas.; \$ USA 29.00

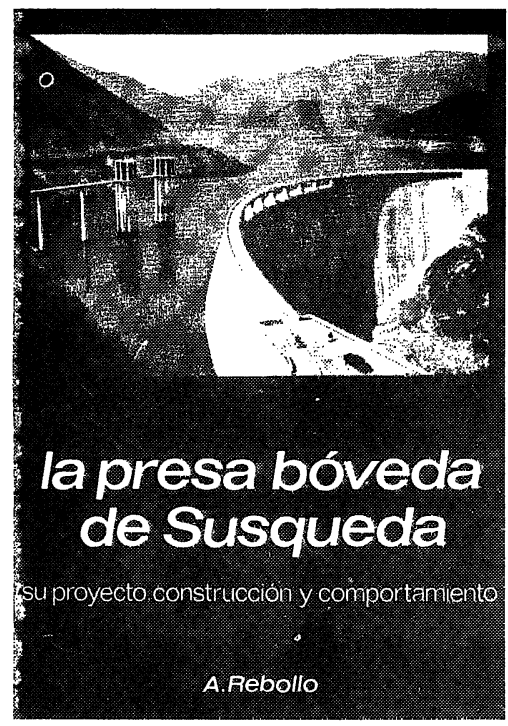

La presa bóveda de Susqueda

A. Rebollo,

Dr. Ingeniero de Caminos

El esfuerzo del constructor de presas se sitúa por su pretensión de perennidad, a contracorriente de las tendencias de la civilizacion actual, caracte rizada por ic fungible. Pueden evocarse las 10.000 grandes presas en funcionamiento o en construcción que estan envejeciendo y reclaman ios cuidados gerontoiógicos para mantener ' $y$ perieccionar su servicio y garantizar su inalienable pretension de perennilad. En la medida en que iodas nuevas rieras, grandes o pequenas, son portadoras de resgos ecologicos r. a veces, catastroficos, que aumentan con el chvejecimiento, la gerontologia de de Arturo Rebolio en emplazo. La accion aceiantada a seguir para ro lo que aro marea un camino a seguir para ros los que arman su bropia obra con

Un volumen encuadernado en cartonè plastificado con tomo de tela, de $18: 24,5 \mathrm{~cm}$, compuesto de 408 páginas, 330 figuras y fotografias y 39 iabias.

Precios: 1.700 ptas.; extranjero, S USA 24.00. 